\title{
Therapies Available for the Treatment of Fungal Nail Infection
}

Pavan Nayak*

Department of Biology, University of California, Santa Cruz, USA

\begin{abstract}
Onychomycosis, a fungal infection caused by dermatophyte fungi such as $T$. rubrum and non-dermatophyte fungi which affect about $2-8 \%$ of people worldwide. Treatment of onychomycosis is hampered owing to poor systemic circulation into the nail apparatus and highly compact structure of nail plate that limits the topical delivery of drugs. Basically, two types of therapies are discussed in this review article, which are traditional and device based therapies. Traditional therapy includes surgical, topical and systemic treatments of onychomycosis. Device-based therapy is a non-invasive treatment which includes lontophoresis, Ultrasound, Photodynamic, and Laser treatments.
\end{abstract}

Keywords: Nail infection; Onychomycosis; Iontophoresis; Topical; Non-invasive

\section{Introduction}

Onychomycosis, a fungal infection caused by dermatophyte fungi such as T. rubrum and non-dermatophyte fungi which affect about $2-8 \%$ of people worldwide [1-3]. Depending on the location of infection and dermatophyte species, four different classifications of onychomycosis are possible: 1) White superficial, 2) Candidal, 3) Proximal subungual, and 4) Distal subungual [1]. Symptoms of fungal infection include thickness/brittleness of the nail plate, with possible discoloration, inflammation, and may lead to breaking off pieces of the nail plate [4]. In addition, Onychomycosis may cause further complications in those with compromised immune system diseases, obesity, or diabetes [5-8].

Treatment of onychomycosis is hampered owing to poor systemic circulation into the nail apparatus and highly compact structure of nail plate that limits the topical delivery of drugs. Two classes of treatments are currently available: Traditional therapies and devicebased therapies [7,9-11]. Traditional treatment includes systemic and topical administration of drugs and partial/complete surgical removal of the nail plate, while biophysical treatments consist of non-invasive therapies such as laser therapy, photodynamic therapy, ultrasoundbased therapy, and Iontophoresis $[1,10,12,13]$. Basically, traditional therapies are dependent upon location and severity of infection progression. Topical treatment is only used when the fungal infection is limited in a few fingers or toes, whereas systemic treatments are used when the infection is spread through multiple digits $[1,6]$. Surgical therapy is performed in chronic conditions when most of the nail plate is affected by fungi.

\section{Traditional Therapies}

\section{Complete/partial nail avulsion}

Nail avulsion or the removal of the nail plate is mainly carried out to determine the spread of infection in the nail bed, separate the infected nail plate from adjacent tissue and treat a fungal infection that has spread to nearby tissue areas. As surgical treatment is the most invasive treatment for onychomycosis, it leads to some severe complications such as hematoma and nail warping/distortion [14-18]. Two types of avulsion are prevalent in clinical treatment today: chemical avulsion and surgical avulsion. Chemical avulsion uses keratolytic compounds, generally salicylic acid or urea, combined with antifungal drugs to dissolve the binding between the nail plate and nail bed [1]. A medical professional may also use phenol or sodium hydroxide to conduct a matricectomy to remove the nail matrix completely. Although chemical avulsion is less painful than surgical avulsion, it is a very tedious process because the bonds take more time to dissolve than to physical removal of the nail plate. In the case of surgical avulsion, the affected area is anesthetized and then treated according to the type of infection [14]. It has been reported more successful with a combination of topical drug treatment. There are two main categories of surgical nail avulsion: distal surgical avulsion and proximal surgical avulsion. In the case of distal avulsion, an instrument is wedged under the nail plate at the distal side and the nail plate is lifted and separated from the nail bed at the distal end. After the instrument is placed under the nail on the proximal edge, the nail plate is separated completely. In a proximal avulsion, the instrument is inserted in the proximal edge initially and the proximal side of the nail is lifted off on the nail bed. The instrument is then inserted all the way through the nail until it reaches the distal end and the plate is removed completely from the nail matrix [14]. Since it is such a drastic procedure, with high patient non-compliance and higher recurrence rates, nail avulsions are usually reserved for persistent cases of onychomycosis.

\section{Topical treatment}

Topical treatment has shown to be very effective in the treatment of smaller localized infections such as white superficial onychomycosis [1]. To enhance the effectiveness of topical therapy, it is often combined with systemic treatments to fully eliminate the source of infection at the nail. In addition, nail permeability enhancers are widely used to increase drug permeability across nail plate. Chemical permeation enhancers improve the permeation of drugs working with a different mechanism of actions such as some of them work as a moisturizer that enhances the water holding capacity of the nail plate and some break the disulfide bonds of keratin protein. The permeability of drug can also be affected by its $\mathrm{pH}$, charge, solubility, molecular size, among other aspects [10]. Two effective topical antifungal drugs in use for onychomycosis treatment today are Ciclopirox and Amorolfine. Ciclopirox, a drug in the hydroxyl pyridine family and an antibacterial, works primarily by blocking enzymes that rely on metals such as iron

*Corresponding author: Pavan Nayak, Department of Biology, University of California, Santa Cruz, USA, Tel: 408-624-0334; E-mail: pavanknayak@gmail.com

Received July 04, 2016; Accepted July 06, 2016; Published July 13, 2016

Citation: Nayak P (2016) Therapies Available for the Treatment of Fungal Nail Infection. J Bioequiv Availab 8: 208-210. doi:10.4172/jbb.1000296

Copyright: (c) 2016 Nayak P. This is an open-access article distributed under the terms of the Creative Commons Attribution License, which permits unrestricted use, distribution, and reproduction in any medium, provided the original author and source are credited. 
[1]. Amorolfine, a morpholine drug, works through the inhibition of ergosterol biosynthesis, effectively changing fungal cell membrane permeability and interrupting key metabolic processes [1]. Side effects for Amorolfine may include itching or burning sensations, as well as pain and irritation [1]. Both Amorolfine and Ciclopirox are only approved in Europe currently, are available in a nail lacquer form, and are mainly used for milder onychomycosis cases such as distal subungual or lateral subungual [1].

\section{Oral and systemic treatment}

Systemic therapy (Oral) is basically used when people are affected by fungal infection in more than 3-4 nails [6]. However, oral drugs have the downsides of decreased success rates and increased treatment length due to poor systemic circulation in the fingers and toes, and low systemic bioavailability of drugs. In addition, long-term systemic or oral therapy may cause some severe side effects such as causing cardiac, liver, and gastric issues.

Oral drugs are classified mainly into two classes: Azoles, and allylamines. These two classes basically work by similar mechanisms of action: disruption of ergosterol biosynthesis, leading to decreased fungal cell membrane integrity, and perturbation of cellular processes $[1,6]$. Their difference lies in the specific enzyme being inhibited: Azoles inhibit lanosterol 14 a-demethylase, while allylamines inhibit squalene epoxidase. The main drugs within the azole category include ketoconazole, itraconazole, and ketoconazole. The primary allylamine drug used in onychomycosis treatment is terbinafine. Griseofulvin works by a different mechanism of action than azoles and allylamines drugs. It binds with microtubule of fungal cell and inhibits the mitosis process $[1,6]$.

\section{Device-based Therapies}

Device-based therapies are non-invasive treatments for the onychomycosis. These therapies include Iontophoresis, Ultrasound, Photodynamic, and Laser treatments. Although device based therapies have higher success rate than traditional therapies, they are not patient compliance and medical expert is required to perform them $[1,17,19]$.

Iontophoresis is a direct current based technique which induces the repulsion force between the electrode and charged drug. The application of iontophoresis is still being investigated to improve the permeation of drugs across the nail plate. Previous literature reported that iontophoresis technique was able to deliver a significant amount of ciclopirox olamine and terbinafine hydrochloride across the nail plate compared to passive delivery. Recently, iontophoresis was used to deliver terbinafine from hyponychium region to ventral layer of the nail plate and other parts of the nail apparatus [3]. Compared to other drug delivery techniques, Iontophoresis can control the flux rate of the drug across the nail plate by application of current. In the case of iontophoresis, physiochemical properties of drug and formulation can also affect the permeation of drug which are current density, vehicle $\mathrm{pH}$, and ionic strength of the buffer. If the current density is increased, the nail drug delivery is also improved as well. Similarly, an optimal buffer ionic strength and vehicle $\mathrm{pH}$ must be sustained in order to obtain the best permeation possible through the nail plate [2,3,20-24].

Ultrasound, a newer addition to the device based onychomycosis strategy, works by pulsing drugs through nail plate using sound waves. Using canine hoof membrane, a drug has been shown to pass through the nail at a rate of $1.5 \times$ faster than simple passive permeation [25]. Ultrasound technique is required to explore for ungual and transungual delivery of drugs.
Photodynamic therapy is a new emerging therapy which stops or kills the fungal infection successfully [26-30]. It works through the process of creating singlet oxygen as a result of excitation of photosensitizing agents by visible light. Singlet oxygen then causes fungal cell death. Two types of photosensitizing agents for this application are currently available, aminolevulinic acid and methyl ester methyl aminolevulinate. Both of these agents are precursors for the creation of protoporphyrin IX which, under red light, is able to penetrate deeper into the nail plate. To increase the permeability of these photosensitizing agents, urea cream is added prior to treatment [31-33]. Adverse side effects of this treatment can include blistering and swelling, as well as bruising of the treated area [31]. Post-therapy considerations include restraining from exposure of the treated area to bright light and avoiding activities that cause heating of the treated area (showers, exercise, etc.) for 2 days after treatment.

Another recent development is laser therapy, which works primarily by the process of photo thermolysis $[1,7]$. Though traditional laser treatments used carbon dioxide, other laser types are currently under study. The laser works by transmitting pulses of radiation into the affected area and is selectively absorbed and stored as heat energy by the fungal cells contained within the infection $[1,7]$. Adverse effects of laser treatment generally stem from higher laser frequency use, which can cause healthy tissue damage, or from overly frequent use, which can cause lowered amounts of collagen in healthy human tissue.

\section{Conclusion}

While there is no one-size-fits-all treatment for onychomycosis, hierarchical levels of treatment are available for different sites and types of infection. For milder onychomycosis cases, typically topical and/ or oral treatments are used. Though oral therapies are more effective than topical therapies, their wide range of severe side effects, and poor circulation in the affected area cause this treatment's usage to be limited in practice. While device based therapies offer a promising solution to fungal infections, they must be further tested and researched before these therapies can be fully brought to market. In the most chronic cases of onychomycosis, either surgical or chemical based nail avulsions are used in combination with a topical therapy. However, high recurrence rates and high patient dropout rates make avulsions a much more drastic measure.

\section{References}

1. Kushwaha A, Murthy RN, Murthy SN, Elkeeb R, Hui X, et al. (2015) Emerging therapies for the treatment of ungual onychomycosis. Drug Dev Ind Pharm 41: 1575-1581.

2. Kushwaha A, Jacob M, Shiva Kumar HN, Hiremath S, Aradhya S, et al. (2015) Trans-ungual delivery of itraconazole hydrochloride by iontophoresis. Drug Dev Ind Pharm 41: 1089-1094.

3. Kushwaha A, Shivakumar HN, Murthy SN (2016) lontophoresis for drug delivery into the nail apparatus: exploring hyponychium as the site of delivery. Drug Dev Ind Pharm 19: 1-5.

4. Elewski BE (1998) Onychomycosis: pathogenesis, diagnosis, and management Clin Microbiol Rev 11: 415-429.

5. Gupta AK, Humke S (2000) The prevalence and management of onychomycosis in diabetic patients. Eur J Dermatol 10: 379-384.

6. Gupta AK, Paquet M, Simpson FC (2013) Therapies for the treatment of onychomycosis. Clin Dermatol 31: 544-554.

7. Gupta A, Simpson F (2012) Device-based therapies for onychomycosis treatment. Skin Therapy Lett 17: 4-9.

8. Juluri A, Peddikotla P, Repka MA, Murthy SN (2013) Transdermal iontophoretic delivery of propofol: a general anaesthetic in the form of its phosphate salt. $J$ Pharm Sci 102: 500-507. 
9. Murthy SN, Maibach HI (2013) (eds.) Topical nail products and ungual drug delivery. CRC Press, Taylor \& Francis Group.

10. Shivakumar HN, Juluri A, Desai BG, Murthy SN (2012) Ungual and transungual drug delivery. Drug Dev Ind Pharm 38: 901-911.

11. Manda P, Angamuthu M, Hiremath SR, Raman V, Murthy SN (2014) lontophoretic drug delivery for the treatment of scars. J Pharm Sci 103: 16381642.

12. Murthy SN (2013) lontophoresis for treating nail diseases. Ther Deliv 4: 647650.

13. Juluri A, Murthy SN (2014) Transdermal iontophoretic delivery of a liquid lipophilic drug by complexation with an anionic cyclodextrin. J Control Release 189: 11-18.

14. Pandhi D, Verma $P(2012)$ Nail avulsion: indications and methods (surgical nail avulsion). Indian J Dermatol Venereol Leprol 78: 299-308.

15. Albom MJ (1977) Surgical gems: avulsion of a nail plate. J Dermatol Surg Oncol 3: 34-35.

16. Scher RK (1987) Nail surgery. Clin Dermatol 5: 135-142.

17. Manda P, Hargett JK, Vaka SR, Repka MA, Murthy SN (2011) Delivery of cefotaxime to the brain via intranasal administration. Drug Dev Ind Pharm 37: 1306-1310.

18. Juluri A, Popescu C, Zhou L, Murthy RN, Gowda VK, et al. (2016) Taste Masking of Griseofulvin and Caffeine Anhydrous Using Kleptose Linecaps DE17 by Hot Melt Extrusion. AAPS PharmSciTech 17: 99-105.

19. Loria MJ, White SW, Robbins SA, Salmeto AL, Hymel KA, et al. (2013) Brainderived neurotrophic factor response in vulnerable and resilient genetic lines in the chick anxiety-depression model. Behav Brain Res 245: 29-33.

20. Nair AB, Kim HD, Chakraborty B, Singh J, Zaman M, et al. (2009) Ungual and trans-ungual iontophoretic delivery of terbinafine for the treatment of onychomycosis. J Pharm Sci 98: 4130-4140.

21. Nair AB, Vaka SR, Sammeta SM, Kim HD, Friden PM, et al. (2009) Transungual iontophoretic delivery of terbinafine. J Pharm Sci 98: 1788-1796.
22. Manda P, Kushwaha AS, Kundu S, Shivakumar HN, Jo SB, et al. (2016) Delivery of ziconotide to cerebrospinal fluid via intranasal pathway for the treatment of chronic pain. J Control Release 224: 69-76.

23. Manda P, Sammeta SM, Repka MA, Murthy SN (2012) lontophoresis across the proximal nail fold to target drugs to the nail matrix. J Pharm Sci 101: 23922397.

24. Yang Y, Manda P, Pavurala N, Khan MA, Krishnaiah YS (2015) Development and validation of in vitro-in vivo correlation (IVIVC) for estradiol transdermal drug delivery systems. J Control Release 210: 58-66.

25. Mididoddi P, Upadhye S, Raymond C, Church C, Davis S, et al. (2006) Influence of etching and ultrasound on the permeability of ciclopirox through the human nail. AAPS San Antonio 8: R6114.

26. Becker C, Bershow A (2013) Lasers and photodynamic therapy in the treatment of onychomycosis: a review of the literature. Dermatol Online J 19: 19611.

27. Harris F, Pierpoint L (2012) Photodynamic therapy based on 5-aminolevulinic acid and its use as an antimicrobial agent. Med Res Rev 32: 1292-1327.

28. Smijs TG, van der Haas RN, Lugtenburg J, Liu Y, de Jong RL, et al. (2004) Photodynamic treatment of the dermatophyte Trichophyton rubrum and its microconidia with porphyrin photosensitizers. Photochem Photobiol 80: 197202.

29. Xu X, Al-Ghabeish M, Rahman Z, Krishnaiah YS, Yerlikaya F, et al. (2015) Formulation and process factors influencing product quality and in vitro performance of ophthalmic ointments. Int J Pharm 493: 412-425.

30. Juluri A, Modepalli N, Jo S, Repka MA, Shivakumar HN, et al. (2013) Minimally invasive transdermal delivery of iron-dextran. J Pharm Sci 102: 987-993.

31. Gupta AK, Simpson FC (2012) Medical devices for the treatment of onychomycosis. Dermatol Ther 25: 574-581.

32. Popescu C, Manda P, Juluri A, Janga KY, Cidda M, et al. (2015) Enhanced dissolution efficiency of zaleplon solid dispersions via modified ß-cyclodextrin molecular inclusion complexes. J Pharm Pharm Sci 1: 12-21.

33. Köllmer M, Popescu C, Manda P, Zhou L, Gemeinhart RA (2013) Stability of benzocaine formulated in commercial oral disintegrating tablet platforms. AAPS PharmSciTech 14: 1333-1340. 